\title{
PERMANENCE AND CONVERGENCE IN MULTI-SPECIES COMPETITION SYSTEMS WITH DELAY
}

\author{
JIANHONG WU AND XIAO-QIANG ZHAO
}

(Communicated by Hal L. Smith)

\begin{abstract}
The permanence and global attractivity of positive equilibria are obtained for some multi-species Kolmogorov competition models with delay by embedding the system into a larger cooperative system with delay and then appealing to the theory of monotone dynamical systems.
\end{abstract}

It is now well-known that the theory of monotone dynamical systems provides a powerful tool for the study of the global dynamics of multi-species cooperative systems, and that a two-species competition system can be transformed into a cooperative system by a simple change of variable (see [4] and [7]-[9] for details). The purpose of this short note is to show that in some situations it is also possible to apply the theory of monotone dynamical systems to deal with $n$-species (with $n>2$ ) competition systems. Our approach is to embed the $n$-species competitive model into a $2 n$-cooperative system under quite general conditions. We will establish the existence and global attractivity of a positive equilibrium for the original $n$-species competition model. For a similar embedding approach and upperand lower-solution method, we refer to the recent paper [6] on nonlinear parabolic systems with time delays.

Let $r \geq 0$ be given, and let $C^{n} \equiv C\left([-r, 0], R^{n}\right)(n \geq 1)$ be the Banach space of continuous mappings from $[-r, 0]$ into $R^{n}$, equipped with the usual uniform norm $\|\phi\|=\sup \{|\phi(s)| ;-r \leq s \leq 0\}$. In what follows, we will use $\hat{x}$ to denote the constant mapping in $C^{n}$ with the value $x \in R^{n}$. Let

$$
R_{+}^{n}=\left\{x=\left(x_{1}, \cdots, x_{n}\right) ; x_{i} \geq 0 \text { for all } 1 \leq i \leq n\right\}
$$

and

$$
C_{+}^{n}=\left\{\phi=\left(\phi_{1}, \cdots, \phi_{n}\right) \in C^{n} ; \phi(s) \in R_{+}^{n} \text { for all }-r \leq s \leq 0\right\} .
$$

Then $\left(R^{n}, R_{+}^{n}\right)$ and $\left(C^{n}, C_{+}^{n}\right)$ are strongly ordered Banach spaces with the cones $R_{+}^{n}$ and $C_{+}^{n}$, respectively. As usual, if $x(\cdot):[-r, \alpha) \rightarrow R^{n}$ is a continuous function, $\alpha>0$ and $t \in[0, \alpha)$, then $x_{t} \in C^{n}$ is defined by $x_{t}(s)=x(t+s),-r \leq s \leq 0$. We

Received by the editors November 15, 1996

1991 Mathematics Subject Classification. Primary 34K15, 58F25, 92D25.

Key words and phrases. Delayed competition systems, monotone semiflow, global attractivity.

The first author's research was supported in part by NSERC and by the Alexander von Humboldt Foundation.

The second author is on leave from the Institute of Applied Mathematics, Academia Sinica, Beijing 100080, China. Research supported in part by the NSF of China.

(C)1998 American Mathematical Society 
consider the following system of functional differential equations:

$$
\frac{d u_{i}(t)}{d t}=u_{i}(t) f_{i}\left(u_{i}(t), u_{t}\right), \quad 1 \leq i \leq n,
$$

where $u(t)=\left(u_{1}(t), \cdots, u_{n}(t)\right)$ and $f_{i}(\cdot, \cdot): R \times C^{n} \rightarrow R$ is locally Lipschitz continuous for $1 \leq i \leq n$. We further assume $f$ satisfies the following conditions:

(C1) For each $1 \leq i \leq n$ and for any given $\phi \in C_{+}^{n}, f_{i}\left(u_{i}, \phi\right)$ is strictly decreasing for $u_{i} \in R_{+}$; and for each $1 \leq i \leq n$ and for any given $u_{i} \in R_{+}, f_{i}\left(u_{i}, \phi\right)$ is decreasing for $\phi \in C_{+}^{n}$.

(C2) There exists $u^{*}=\left(u_{1}^{*}, \cdots, u_{n}^{*}\right) \in i n t R_{+}^{n}$ such that for each $1 \leq i \leq n$, $f_{i}(0, \hat{0})>0, f_{i}\left(u_{i}^{*}, \hat{0}\right)=0$ and $f_{i}\left(0, \widehat{u^{*}}\right)>0$.

(C3) The algebraic equation $F(u, v)=G(u, v)=0$ admits at most one (componentwise) positive solution $(u, v) \in R^{2 n}$, where $F(u, v)=\left(f_{1}\left(u_{1}, \hat{v}\right), \cdots, f_{n}\left(u_{n}, \hat{v}\right)\right)^{T}$ and $G(u, v)=\left(f_{1}\left(v_{1}, \hat{u}\right), \cdots, f_{n}\left(v_{n}, \hat{u}\right)\right)^{T}$.

$(\mathrm{C} 1)$ is the standard assumption for competition. Roughly speaking, (C2) requires that the competitive system without delayed competition terms possesses a positive equilibrium and the collective effect of instantaneous competition is stronger than that of delayed competition (at least near the aforementioned positive equilibrium). (C3) is the standing assumption for our embedding approach to be applicable. Illustrative examples will be given later.

We will need the following elementary result:

Lemma 1. Consider the scalar autonomous equation

$$
\frac{d u}{d t}=u f(u)
$$

where $f: R \rightarrow R$ is locally Lipschitz. Assume that

(i) $f(0)>0$ and $f(u)$ is strictly decreasing for $u \geq 0$;

(ii) there exists $K>0$ such that $f(u) \leq f(K)<0$ for all $u \geq K$.

Then equation (2) admits a unique positive equilibrium which is globally attractive with respect to positive initial values.

Our main result is the following.

Theorem 1. Assume that (C1) and (C2) hold. Then system (1) admits a positive equilibrium and is permanent, i.e., there exist $\eta>0$ and $M>0$ such that for any $\phi \in C_{+}^{n}$ with $\phi(0) \gg 0$ (i.e., $\phi(0) \in \operatorname{int}\left(R_{+}^{n}\right)$ ), there is $T=T(\phi)>0$ such that $\eta \leq u_{i}(t, \phi) \leq M$ for all $t \geq T$ and $1 \leq i \leq n$. If, in addition, (C3) holds, then system (1) admits a unique positive equilibrium which is globally attractive for $\phi \in C_{+}^{n}$ with $\phi(0) \gg 0$.

Proof. Let $e$ be the vector in $R^{n}$ with all components equal to 1 . Since

$$
\lim _{\epsilon \rightarrow 0} f_{i}\left(0, \widehat{u^{*}}+\epsilon \hat{e}\right)=f_{i}\left(0, \widehat{u^{*}}\right)>0, \quad 1 \leq i \leq n,
$$

we can choose $\epsilon_{0}>0$ such that $f_{i}\left(0, \widehat{u^{*}}+\epsilon_{0} \hat{e}\right)>0$ for all $1 \leq i \leq n$. By $(\mathrm{C} 1),(\mathrm{C} 2)$ and Lemma 1 , for each $1 \leq i \leq n, u_{i}=u_{i}^{*}>0$ is a globally attractive equilibrium in $R_{+} \backslash\{0\}$ of the scalar equation

$$
\frac{d u_{i}}{d t}=u_{i} f_{i}\left(u_{i}, \hat{0}\right),
$$


and the scalar equation

$$
\frac{d u_{i}}{d t}=u_{i} f_{i}\left(u_{i}, \widehat{u^{*}}+\epsilon_{0} \hat{e}\right)
$$

admits a unique positive equilibrium $\underline{u}_{i}^{*}$ which is globally attractive in $R_{+} \backslash\{0\}$.

For any $\phi \in C_{+}^{n}$, let $[0, \beta)$ be the maximal interval of existence of the solution $u(t, \phi)$ of (1) subject to the initial condition $u_{0}=\phi$. By [8, Theorem 5.2.1], $u(t, \phi) \geq$ 0 in $R^{n}$, and hence $u_{t}(\phi) \in C_{+}^{n}$ for all $t \in[0, \beta)$. Furthermore, if $\phi_{i}(0)>0$ for some $1 \leq i \leq n$, then $u_{i}(t, \phi)>0$ for $t \in[0, \beta)$. Let $u(t, \phi)=\left(u_{1}(t), \cdots, u_{n}(t)\right)$; then for each $1 \leq i \leq n, u_{i}(t)$ satisfies

$$
\frac{d u_{i}(t)}{d t}=u_{i}(t) f_{i}\left(u_{i}(t), u_{t}\right) \leq u_{i} f_{i}\left(u_{i}(t), \hat{0}\right), \quad t \in[0, \beta) .
$$

Then, by the standard comparison theorem,

$$
u_{i}(t) \leq \bar{u}_{i}(t), \quad t \in[0, \beta), \quad 1 \leq i \leq n,
$$

where $\bar{u}_{i}(t)$ is the unique solution of (3) with $\bar{u}_{i}(0)=u_{i}(0)=\phi_{i}(0)$. Therefore, the global existence of $\bar{u}_{i}(t)$ on $[0, \infty)(1 \leq i \leq n)$ implies that $\beta=\infty$. Let $\bar{u}(t)=\left(\bar{u}_{1}(t), \cdots, \bar{u}_{n}(t)\right), t \geq 0$. For any given $\phi \in C_{+}^{n}$ with $\phi(0) \gg 0$, by (6) and the fact that $\lim _{t \rightarrow \infty} \bar{u}_{i}(t)=u_{i}^{*}$, there exists $T_{1}=T_{1}(\phi)>0$ such that

$$
0 \ll u(t, \phi) \leq \bar{u}(t) \leq u^{*}+\epsilon_{0} e, \quad \text { for all } t \geq T_{1} .
$$

Therefore

$$
\hat{0} \ll u_{t}(\phi) \leq \widehat{u^{*}}+\epsilon_{0} \hat{e}, \quad \text { for all } t \geq T_{1}+r=T_{2} .
$$

Let $U_{i}(t)=u_{i}\left(t+T_{2}\right), t \geq 0$. Then $U(t)=\left(U_{1}(t), \cdots, U_{n}(t)\right)$ satisfies

$$
\frac{d U_{i}(t)}{d t}=U_{i}(t) f_{i}\left(U_{i}(t), U_{t}\right) \geq U_{i}(t) f_{i}\left(U_{i}(t), \widehat{u^{*}}+\epsilon_{0} \hat{e}\right), \quad t \geq 0,1 \leq i \leq n .
$$

Using the standard comparison theorem again, we get

$$
U_{i}(t) \geq \underline{u}_{i}(t), \quad t \geq 0,1 \leq i \leq n,
$$

where $\underline{u}_{i}(t)$ is the unique solution of equation (4) with $\underline{u}_{i}(0)=U_{i}(0)=u_{i}\left(T_{2}\right)>$ $0,1 \leq i \leq n$. As $\lim _{t \rightarrow \infty} \underline{u}_{i}(t)=\underline{u}_{i}^{*}>0,1 \leq i \leq n$, we conclude that for fixed $0<\eta<\min _{1 \leq i \leq n} \underline{u}_{i}^{*}$, there exists $T_{3}=T_{3}(\phi)>0$ such that

$$
U_{i}(t) \geq \underline{u}_{i}(t) \geq \underline{u}_{i}^{*}-\eta, \quad \text { for all } t \geq T_{3} .
$$

By (7) and (9), it follows that

$$
0<\underline{u}_{i}^{*}-\eta \leq u_{i}(t) \leq u_{i}^{*}+\epsilon_{0}, \quad \text { for all } t \geq T=T_{3}+T_{2}, \quad 1 \leq i \leq n .
$$

Therefore, system (1) is permanent. It is easy to employ the above argument to obtain the permanence of the following autonomous system of ordinary differential equations:

$$
\frac{d u_{i}}{d t}=u_{i} f_{i}\left(u_{i}, \hat{u}\right), \quad 1 \leq i \leq n .
$$

Therefore, the semiflow $T_{0}(t): R_{+}^{n} \rightarrow R_{+}^{n}$ generated by (10) is compact for each $t>0$, point dissipative in $R_{+}^{n}$ and uniformly persistent with respect to $\partial R_{+}^{n}$. By an index theorem in [5, Section 19.3] (see also [10, Theorem 2.4] for infinite dimensional semiflows), $T_{0}(t)$ has an equilibrium in $\operatorname{int}\left(R_{+}^{n}\right)$ and hence system (1) has a positive equilibrium. 
To prove the global attractivity of positive equilibrium under the additional condition (C3), we consider the following extended delayed system in $R^{2 n}$ :

$$
\left\{\begin{array}{l}
\frac{d u_{i}(t)}{d t}=u_{i} f_{i}\left(u_{i}(t), v_{t}\right), \\
\frac{d v_{i}(t)}{d t}=v_{i} f_{i}\left(v_{i}(t), u_{t}\right), \quad 1 \leq i \leq n .
\end{array}\right.
$$

Again by [8, Theorem 5.2.1], $X=C_{+}^{2 n}=C_{+}^{n} \times C_{+}^{n}$ is positively invariant for (11). By an argument similar to that in the proof of the permanence of system (1), we can show that system (11) is permanent, i.e., there exist $\eta>0$ and $M>0$ such that for any $\phi \in C_{+}^{2 n}$ with $\phi(0) \gg 0$ in $\left(R^{2 n}, R_{+}^{2 n}\right)$, there is $T=T(\phi)>0$ such that the unique solution $(u(t, \phi), v(t, \phi))=\left(u_{1}(t, \phi), \cdots, u_{n}(t, \phi), v_{1}(t, \phi), \cdots, v_{n}(t, \phi)\right)$ of system (11) with $\left(u_{0}(\phi), v_{0}(\phi)\right)=\phi$ satisfies $\eta \leq u_{i}(t, \phi) \leq M$ and $\eta \leq v_{i}(t, \phi) \leq$ $M$ for all $t \geq T$ and $1 \leq i \leq n$. Let

$$
\partial X_{0}=\left\{\phi \in C_{+}^{2 n} ; \text { there exists some } 1 \leq i \leq 2 n \text { such that } \phi_{i}(0)=0\right\}
$$

and $X_{0}=X \backslash \partial X_{0}$. Let $T(t): X \rightarrow X$ be defined by $T(t) \phi=\left(u_{t}(\phi), v_{t}(\phi)\right), \phi \in$ $X$. Then $T(t): X \rightarrow X$ is a continuous semiflow with $T(t): X_{0} \rightarrow X_{0}$ and $T(t): \partial X_{0} \rightarrow \partial X_{0}$ for $t \geq 0$. Moreover, by [2, Corollary 3.6.2], $T(t): X \rightarrow X$ is compact for each $t \geq r$. Clearly, $T(t): X \rightarrow X$ is point dissipative and uniformly persistent with respect to $\partial X_{0}$. By [3, Theorem 3.2], there exists a global attractor $A_{0}$ in $X_{0}$ relative to strongly bounded sets in $X_{0}$.

Let $P=C_{+}^{n} \times\left(-C_{+}^{n}\right)$. Then $\left(C^{2 n}, P\right)$ is a strongly ordered Banach space with the cone $P$. By a change of variables $w_{i}=-v_{i}, 1 \leq i \leq n$, and the comparison theorem ([8, Theorem 5.1.1]), condition (C1) then implies that $T(t): X \rightarrow X$ is a monotone semiflow with respect to the order generated by $P$, i.e., for any $\phi, \psi \in X$ with $\phi \leq \psi$ in $\left(C^{2 n}, P\right)$ we have $T(t) \phi \leq T(t) \psi$ in $\left(C^{2 n}, P\right)$ for all $t \geq 0$. By [4, Theorem 3.1] and condition (C3), the global attractor $A_{0}$ contains only one equilibrium $(\hat{u}, \hat{v})$ and hence, by [4, Theorem 3.3], $(\hat{u}, \hat{v})$ attracts every point $(\phi, \psi) \in X_{0}$. In particular, for any $\phi \in C_{+}^{n}$ with $\phi(0) \gg 0$ in $\left(R^{n}, R_{+}^{n}\right)$, let $u(t, \phi)$ be the unique solution of (1) with $u_{0}(\phi)=\phi$. Then $(\phi, \phi) \in X_{0}$, and hence $T(t)(\phi, \phi)=\left(u_{t}(\phi), u_{t}(\phi)\right) \rightarrow(\hat{u}, \hat{v})$ as $t \rightarrow \infty$. It follows that $\hat{u}=\hat{v}, \hat{u}$ is an equilibrium of system (1) and $u_{t}(\phi) \rightarrow \hat{u}$ as $t \rightarrow \infty$. Consequently, $\lim _{t \rightarrow \infty} u(t, \phi)=u$. This completes the proof.

Corollary 1. Let (C1) and (C2) hold. Assume that for each $1 \leq i \leq n, f_{i}\left(u_{i}, \hat{v}\right)$ is a polynomial of degree one with respect to $n+1$ variables $\left(u_{i}, v_{1}, \cdots, v_{n}\right)$. Then system (1) has a unique positive equilibrium which is globally attractive with respect to $\phi \in C_{+}^{n}$ with $\phi(0) \gg 0$ in $\left(R^{n}, R_{+}^{n}\right)$.

Proof. By Theorem 1, it suffices to verify that condition (C3) holds. By condition (C2), we can choose $\epsilon_{0}>0$ such that

$$
f_{i}\left(0, \widehat{u^{*}}+\epsilon_{0} \hat{e}\right)>0, \quad \text { for all } 1 \leq i \leq n .
$$

Let

$$
\Sigma=\left\{(u, v) \in R^{2 n} ; 0 \leq u_{i} \leq u_{i}^{*}+\epsilon_{0} \text { and } 0 \leq v_{i} \leq u_{i}^{*}+\epsilon_{0} \text { for all } 1 \leq i \leq n\right\} .
$$

We claim that $\Sigma$ is a contracting rectangle for the vector field $(F, G)^{T}$ in $R^{2 n}$. For if $(u, v) \in \Sigma$ with $u_{i}=0$ (or $v_{i}=0$ ), then $F_{i}(u, v)=f_{i}(0, \hat{v}) \geq f_{i}\left(0, \widehat{u^{*}}+\epsilon_{0} \hat{e}\right)>0$ $\left(\right.$ or $\left.G_{i}(u, v)=f_{i}(0, \hat{u}) \geq f_{i}\left(0, \widehat{u^{*}}+\epsilon_{0} \hat{e}\right)>0\right)$; and if $(u, v) \in \Sigma$ with $u_{i}=u_{i}^{*}+\epsilon_{0}$ 
(or $\left.v_{i}=u_{i}^{*}+\epsilon_{0}\right)$, then $F_{i}(u, v)=f_{i}\left(u_{i}^{*}+\epsilon_{0}, \hat{v}\right) \leq f_{i}\left(u_{i}^{*}+\epsilon_{0}, \hat{0}\right)<f_{i}\left(u_{i}^{*}, \hat{0}\right)=0$ (or $\left.G_{i}(u, v)=f_{i}\left(u_{i}^{*}+\epsilon_{0}, \hat{u}\right) \leq f_{i}\left(u_{i}^{*}+\epsilon_{0}, \hat{0}\right)<f_{i}\left(u_{i}^{*}, \hat{0}\right)=0\right)$. It follows that $\Sigma$ is positively invariant for the following system of ordinary differential equations in $R^{2 n}$ :

$$
\left\{\begin{array}{l}
\frac{d u}{d t}=F(u, v), \\
\frac{d v}{d t}=G(u, v) .
\end{array}\right.
$$

By a standard argument using the Brouwer fixed point theorem, there exists an equilibrium $\left(u^{*}, v^{*}\right)$ for $(12)$ in the interior of $\Sigma$. Suppose that, for a contradiction, there is another positive equilibrium $(\bar{u}, \bar{v}) \neq\left(u^{*}, v^{*}\right)$. By our assumption on $f_{i}\left(u_{i}, \hat{v}\right)(1 \leq i \leq n)$, it easily follows that $t(\bar{u}, \bar{v})+(1-t)\left(u^{*}, v^{*}\right)=$ $\left(t \bar{u}+(1-t) u^{*}, t \bar{v}+(1-t) v^{*}\right)$ is also a solution of $F=G=0$ for any $t \in R$, and then there must be a solution belonging to the boundary of $\Sigma$. But this is impossible since $\Sigma$ is contracting. Therefore, there is exactly one positive solution of $F=G=0$. Consequently, condition (C3) holds. This completes the proof.

Remark 1. The above corollary shows that if (C1) and (C2) hold for any LotkaVolterra type competition system with delay, then the system admits a unique globally attractive positive equilibrium. Therefore, the delay is harmless for the global dynamics of the system under $(\mathrm{C} 1)$ and $(\mathrm{C} 2)$.

Remark 2. For the delayed competition system (7.1) discussed in [8, Chapter 5], for each $1 \leq i \leq n$, we have

$$
\begin{aligned}
& f_{i}\left(u_{i}, \phi\right) \\
& =b_{i}\left[1-c_{i i}\left(a_{i} u_{i}+\left(1-a_{i}\right) \int_{-r}^{0} \phi_{i}(s) d v_{i i}(s)\right)-\sum_{j=1, j \neq i}^{n} c_{i j} \int_{-r}^{0} \phi_{j}(s) d v_{i j}(s)\right] .
\end{aligned}
$$

Then $u_{i}^{*}=\frac{1}{a_{i} c_{i i}}, 1 \leq i \leq n$, and hence it is easy to see that the condition $f_{i}\left(0, \widehat{u^{*}}\right)>$ $0(1 \leq i \leq n)$ in $(\mathrm{C} 2)$ is just the condition (7.4) of [8, Chapter 5]. Therefore, Corollary 1 implies [8, Theorem 5.7.5], which was proved in [8] by a contracting rectangles argument.

Remark 3. Gopalsamy in [1] considered the following two-species Lotka-Volterra competition system

$$
\left\{\begin{array}{l}
\frac{d u(t)}{d t}=u(t)\left[\gamma_{1}-a_{1} u(t)-b_{1} \int_{-r}^{0} K_{1}(s) v(t+s) d s\right], \\
\frac{d v(t)}{d t}=v(t)\left[\gamma_{2}-a_{2} \int_{-r}^{0} K_{2}(s) u(t+s) d s-b_{2} v(t)\right],
\end{array}\right.
$$

where $a_{1}, a_{2}, b_{1}, b_{2}, \gamma_{1}$ and $\gamma_{2}$ are positive constants, $r>0$ and $K_{1}(\cdot)$ and $K_{2}(\cdot)$ : $[-r, 0] \rightarrow R$ are non-negative continuous functions with $\int_{-r}^{0} K_{1}(s) d s=1$ and $\int_{-r}^{0} K_{2}(s) d s=1$. Clearly, $u_{1}^{*}=\frac{\gamma_{1}}{a_{1}}, u_{2}^{*}=\frac{\gamma_{2}}{b_{2}}$. Then it easily follows that the condition $f_{i}\left(0, \widehat{u^{*}}\right)>0(1 \leq i \leq 2)$ in $(\mathrm{C} 2)$ is equivalent to $\frac{a_{1}}{a_{2}}>\frac{\gamma_{1}}{\gamma_{2}}>\frac{b_{1}}{b_{2}}$, and hence, Corollary 1 also implies the main theorem in [1].

We conclude with two simple examples of scalar delayed differential equations which are not of Lotka-Volterra type. 
Example 1. Consider the scalar delayed equation

$$
\frac{d u(t)}{d t}=u(t)\left[a-\frac{b u(t)}{m+u(t)}-c u(t-\tau)\right]
$$

where $a, b, c, m$ and $\tau$ are positive constants and $b>a$. A direct calculation shows that $u^{*}=\frac{a m}{b-a}>0$. Moreover, $f\left(0, \widehat{u^{*}}\right)>0$ is equivalent to $b-a>\mathrm{cm}$, and (C3) holds if and only if $c m \neq b-a$. Clearly, (C2) implies (C3). Therefore, by Theorem 1, equation (14) admits a globally attractive positive equilibrium under the condition $b-a>c m$.

Example 2. Consider the scalar delayed equation

$$
\frac{d u(t)}{d t}=u(t)\left[a-b u(t)-c u^{2}(t-\tau)\right]
$$

where $a, b, c$ and $\tau$ are positive constants. A direct calculation shows that $u^{*}=$ $\frac{a}{b}>0$. Moreover, $f\left(0, \widehat{u^{*}}\right)>0$ is equivalent to $b^{2}>a c$, and (C3) holds if and only if either $b^{2} \leq a c$ or $b^{2} \geq \frac{4}{3} a c$. Therefore, by Theorem 1, equation (15) admits a globally attractive positive equilibrium under the condition $b^{2} \geq \frac{4}{3} a c$. It should be mentioned that in this example, $(\mathrm{C} 2)$ doesn't imply (C3).

\section{REFERENCES}

[1] K. Gopalsamy, Time lags and global stability in two-species competition, Bull. Math. Biology, 42(1980), 729-737. MR 84b:92068

[2] J. K. Hale and S.M. Verduyn Lunel, Introduction to Functional Differential Equations, Applied Mathematical Sciences, Vol. 99, Springer-Verlag, New York, 1993. MR 94m:34169

[3] J. K. Hale and P. Waltman, Persistence in infinite-dimensional systems, SIAM J. Math. Anal., 20(1989), 388-395. MR 90b:58156

[4] M. Hirsch, The dynamical systems approach to differential equations, Bull. A.M.S., 11(1984), 1-64. MR 85m:58060

[5] J. Hofbauer and K. Sigmund, The Theory of Evolution and Dynamical Systems, London Math. Society Student Texts, Vol. 7, Cambridge University Press, 1988. MR 91h:92019

[6] C. V. Pao, Dynamics of nonlinear parabolic systems with time delays, J. Math. Anal. Appl., 198(1996), 751-779. MR 97d:35229

[7] H. L. Smith, Systems of ordinary differential equations which generate an order preserving flow, A survey of results, SIAM Review, 30(1988), 87-113. MR 89f:34005

[8] H.L. Smith, Monotone Dynamical Systems, An Introduction to the Theory of Competitive and Cooperative Systems, Mathematical Surveys and Monographs,Vol. 41, A.M.S., Providence, RI, 1995. MR 96c:34002

[9] H. L. Smith and P. Waltman, The Theory of The Chemostat, Cambridge University Press, 1995. MR 96e:92016

[10] X.-Q. Zhao, Uniform persistence and periodic coexistence states in infinite dimensional periodic semiflows with applications, Canad. Appl. Math. Quart., 3(1995), 473-495. MR 96k:58188

Department of Mathematics and Statistics, York University, North York, Canada M3J 1P3

E-mail address: wujh@mathstat.yorku.ca

Current address, X.-Q. Zhao: Department of Mathematics, Arizona State University, Tempe, Arizona 85287

E-mail address: xzhao@math.la.asu.edu 